\title{
Extended $\mathrm{BaZrO}_{3}$ Nanodot Columns in Thick $\mathrm{YB}_{2} \mathrm{Cu}_{3} \mathrm{O}_{7}$ Film
}

\author{
J. Li,* S. Kang,* and A. Goyal*
}

* Metal and Ceramics Division, Oak Ridge National Laboratory, P.O. Box 2008, MS 6116, Oak Ridge, TN 37831

In the development of the second generation coated conductors, various artificial pinning defects have been introduced into superconductors to enhance the critical current density $\left(J_{c}\right)$ for electric power applications. Among these defects, columnar defects were proven to be most effective. We have shown previously that the columnar defects comprised of self-aligned nanodots of $\mathrm{BaZrO}_{3}$ (BZO) incorporated during growth of $\mathrm{YBa}_{2} \mathrm{Cu}_{3} \mathrm{O}_{7}(\mathrm{YBCO})$ films significantly improve the flux pinning properties [1-2]. Even in films as thick as $3 \mu \mathrm{m}$, we have demonstrated that the self-aligned BZO nanodots which are aligned parallel to the $c$-axis of YBCO, nucleate at buffer layer interface and extend to the top of the YBCO film [2]. The superconducting transport properties of these thick films were very good, specially in applied fields.

Cross-section transmission electron microscopy (TEM) was used for microstructural analysis using a Hitachi HF-2000 Microscope with field emission gun (FEG) operated at $200 \mathrm{kV}$ and a Philips CM200 Microscope with FEG operated at $200 \mathrm{kV}$. The BZO nanoparticles have cubic structure (perovskite, $\mathrm{a}_{0}=\mathrm{b}_{0}=\mathrm{c}_{0}=4.193 \AA$ ), which are coherently embedded in YBCO film matrix (orthorhombic structure, $\mathrm{a}_{0}=3.825 \AA, \mathrm{b}_{0}=3.886 \AA, \mathrm{c}_{0}=11.66 \AA$ ) with the lattice mismatches of $+9.6 \%$ for $(100) \mathrm{BZO} /(100) \mathrm{YBCO},+7.9 \%$ for $(010) \mathrm{BZO} /(010) \mathrm{YBCO}$ and $+7.9 \%$ for $(001) \mathrm{BZO} /(003) \mathrm{YBCO}$. Due to the strain field contrast and the Moiré fringes produced by coherency and lattice mismatch, the location and distribution of the BZO nanoparticles pattern can be clearly shown.

Fig 1a is cross-section TEM micrographs for $3.0 \mu \mathrm{m}$ thick YBCO film with BZO nanodots grown epitaxially on rolling-assisted-biaxially-textured-substrates (RABiTS [3]). Fig. 1b shows TEM image with higher magnification for the region of YBCO + BZO in Fig. 1a, showing that the BZO nanoparticles have been self-aligned to form array of periodic long columns along [001] direction of YBCO. Fig. 2 shows that the self-aligned BZO nanodot columns $(<10 \mathrm{~nm}$ in width) extend through the entire cross section of the $3 \mu \mathrm{m}$ thick YBCO film from the bottom of YBCO layer (figure 2a) to the top of the YBCO layer (figure 2b). These microstructure features explain the significantly enhanced critical current density and flux pinning of the YBCO films [2].

References

[1] A. Goyal et al., Supercond. Sci Technol. 18, (2005) 1533.

[2] S. Kang et al., to be published in Science, (2006).

[3] A. Goyal et al., Appl. Phys. Lett. 69, (1996) 1795.

[4] Research sponsored by the USDOE, under contract DE-AC05-00OR22725. 


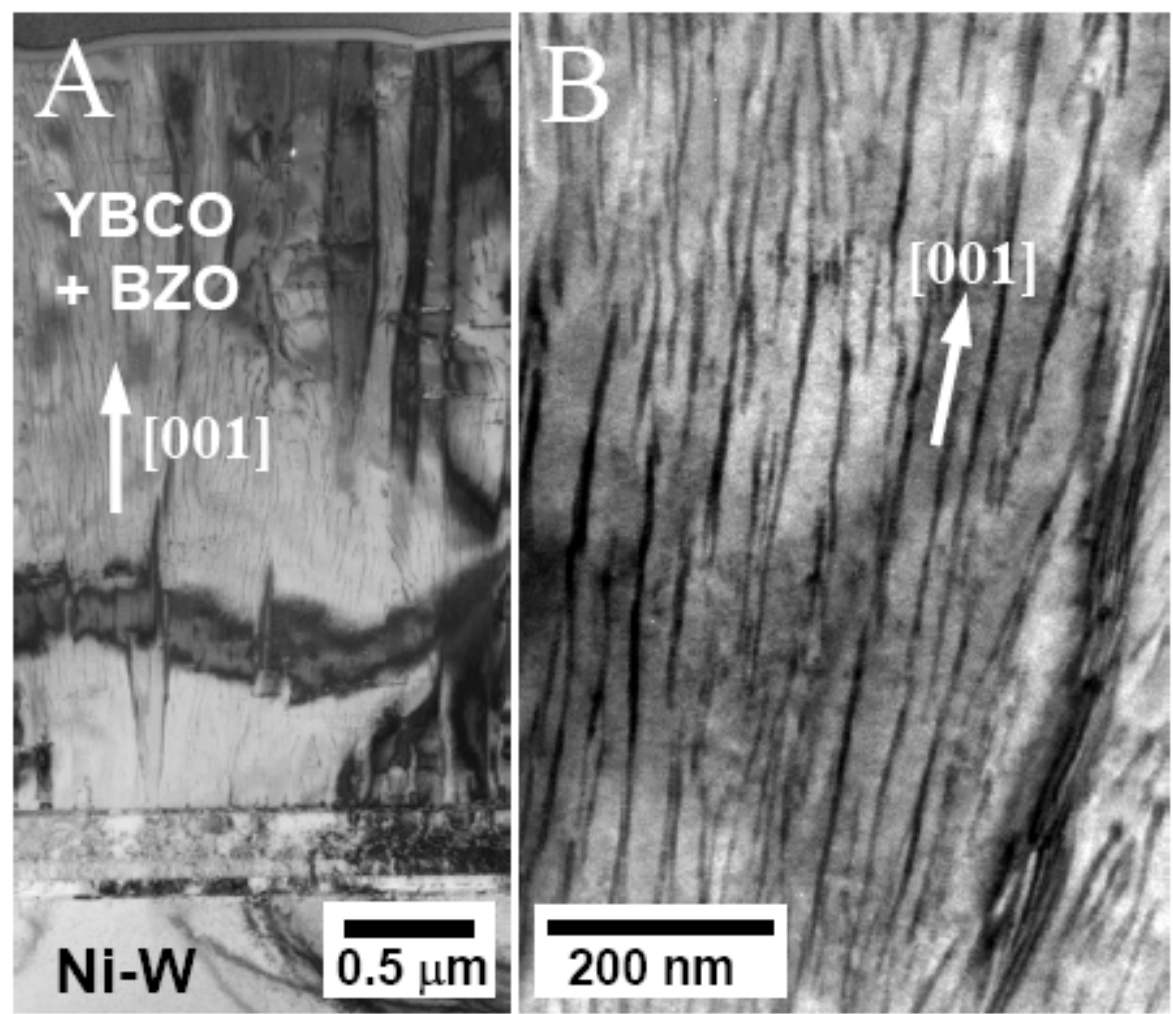

Fig. 1. A. Cross-section TEM micrograph for $3.0 \mu \mathrm{m}$ thick YBCO film with BZO nanodots. B. Selfaligned BZO nanoparticles form array of periodic long columns along [001] direction of YBCO.

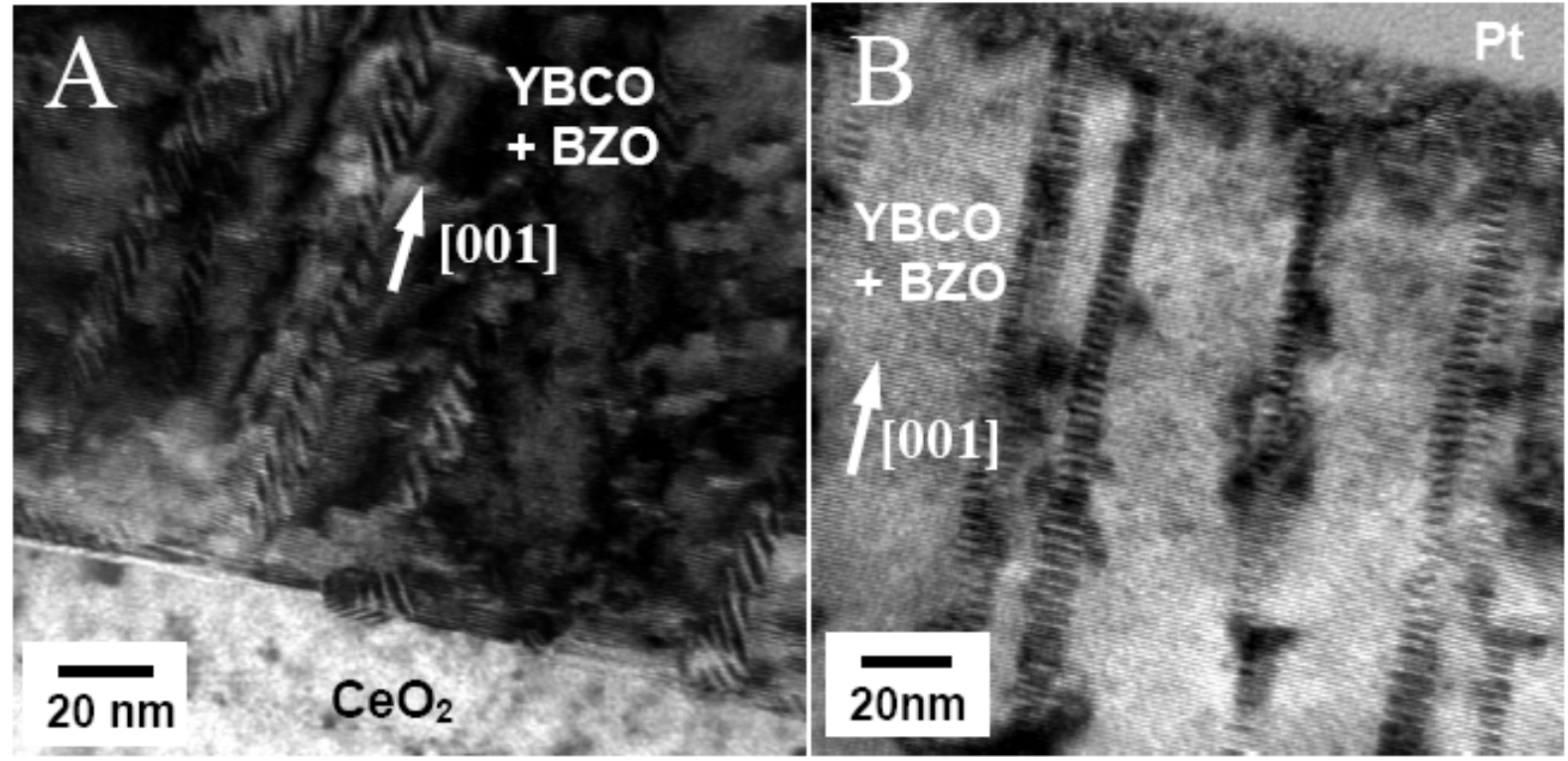

Fig. 2. Moiré fringes provide BZO nanoparticles pattern in YBCO film. Self-aligned BZO nanodot columns were extended through the entire cross section of the $3 \mu \mathrm{m}$ thick YBCO film from the bottom of YBCO layer (A) to the top of the YBCO layer (B). 